\title{
A definitive signal of multiple supersymmetry breaking
}

\author{
Clifford Cheung, ${ }^{a, b}$ Jeremy Mardon, ${ }^{a, b}$ Yasunori Nomura ${ }^{a, b, c}$ and Jesse Thaler $^{d}$ \\ ${ }^{a}$ Berkeley Center for Theoretical Physics, University of California, \\ Berkeley, CA 94720, U.S.A. \\ ${ }^{b}$ Theoretical Physics Group, Lawrence Berkeley National Laboratory, \\ Berkeley, CA 94720, U.S.A. \\ ${ }^{c}$ Institute for the Physics and Mathematics of the Universe, \\ University of Tokyo, Kashiwa 277-8568, Japan \\ ${ }^{d}$ Center for Theoretical Physics, Massachusetts Institute of Technology, \\ Cambridge, MA 02139, U.S.A. \\ E-mail: clifford.cheung@berkeley.edu, jmardon@berkeley.edu, \\ YNomura@lbl.gov, jthaler@jthaler.net
}

ABSTRACT: If the lightest observable-sector supersymmetric particle (LOSP) is charged and long-lived, then it may be possible to indirectly measure the Planck mass at the LHC and provide a spectacular confirmation of supergravity as a symmetry of nature. Unfortunately, this proposal is only feasible if the gravitino is heavy enough to be measured at colliders, and this condition is in direct conflict with constraints from big bang nucleosynthesis (BBN). In this work, we show that the BBN bound can be naturally evaded in the presence of multiple sectors which independently break supersymmetry, since there is a new decay channel of the LOSP to a goldstino. Certain regions of parameter space allow for a direct measurement of LOSP decays into both the goldstino and the gravitino at the LHC. If the goldstino/gravitino mass ratio is measured to be 2, as suggested by theory, then this would provide dramatic verification of the existence of multiple supersymmetry breaking and sequestering. A variety of consistent cosmological scenarios are obtained within this framework. In particular, if an $R$ symmetry is imposed, then the gauge-gaugino-goldstino interaction vertices can be forbidden. In this case, there is no bound on the reheating temperature from goldstino overproduction, and thermal leptogenesis can be accommodated consistently with gravitino dark matter.

KeYwORDS: Cosmology of Theories beyond the SM, Supersymmetry Breaking

ARXIV EPRINT: 1004.4637 


\section{Contents}

1 Introduction $\quad 1$

$2 \quad \mathrm{BBN}$ and the LHC 3

3 Review of Goldstini framework 5

4 Gravitino and Goldstini at colliders $\quad 6$

5 Viable cosmologies $\quad 9$

5.1 Reheating bounds on Goldstini couplings 9

$\begin{array}{ll}5.2 \text { The minimal Goldstini scenario } & 10\end{array}$

5.3 SUSY breaking with $R$ symmetries $\quad 11$

$\begin{array}{ll}5.4 \text { Late decay case } & 12\end{array}$

6 Other LOSPs 12

$\begin{array}{lll}7 & \text { Conclusions } & 14\end{array}$

$\begin{array}{ll}\text { A Infrared-dominated Goldstino production } & 15\end{array}$

\section{Introduction}

At first glance, particle colliders do not appear particularly well-suited to the task of probing the fundamental structure of gravity. Indeed, given the intrinsic energy limitations of present day and future machines, any direct experimental handle on Planck scale physics continues to be a rather remote possibility. Nevertheless, weak scale supersymmetry (SUSY) may provide a unique window into genuinely gravitational physics because SUSY is a symmetry of spacetime.

In particular, if SUSY is realized as a local symmetry, namely supergravity (SUGRA), then there necessarily exists a spin-3/2 superpartner of the graviton: the gravitino. The gravitino has a mass, $F / \sqrt{3} M_{\mathrm{Pl}}$, and couplings to observable sector fields, $\sim 1 / F$, which obey a fixed relationship determined by the Planck scale, $M_{\mathrm{Pl}}$. Because the existence of the gravitino is mandatory, it is an attractive possibility that this state comprises the dark matter of the universe. In this case, the lightest observable-sector supersymmetric particle (LOSP) may be charged, and precision studies of the LOSP decay into the gravitino can provide a robust test of the expected gravitino mass/interaction relation and therefore an indirect measurement of $M_{\mathrm{Pl}}[1,2]$. This would offer compelling evidence for the validity of SUGRA as well as a genuine probe of gravitational physics at particle colliders. 


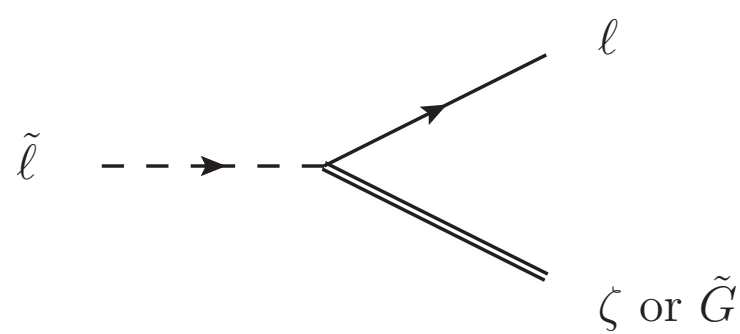

Figure 1. Slepton LOSP decay into a goldstino or a gravitino.

Unfortunately, this most spectacular signal is in direct conflict with big bang nucleosynthesis $(\mathrm{BBN})$. At colliders, $M_{\mathrm{Pl}}$ can only be measured if the gravitino is sufficiently heavy: $m_{3 / 2} \gtrsim O(0.1) m_{\text {LOSP }}$. However, unless the gravitino is sufficiently light, $m_{3 / 2} \lesssim O(1-10 \mathrm{GeV})$, then late-time charged LOSP decays destroy the successful predictions for the abundance of light elements [3-5]. Thus, if the gravitino is to be heavy enough for a successful collider measurement, one must resort to a rather non-standard cosmology in which the thermal history is modified below a temperature of $O(0.1) m_{\text {LOSP }}$. Additionally, thermal leptogenesis [6] - arguably the simplest mechanism for baryogenesis — does not work for the small values of $m_{3 / 2}$ required by the BBN constraint unless the gravitino is extremely light $\left(m_{3 / 2} \lesssim 10 \mathrm{eV}\right)$. This is a consequence of gravitino overproduction from the high temperature plasma $[7,8]$ and constraints from structure formation [9].

It is interesting to note, however, that the BBN bound actually has nothing to do with the gravitino itself - it has to do with the late decaying LOSP injecting energies during or after BBN. Therefore, if there is an additional state to which the LOSP can decay more quickly, then the constraint from BBN may be avoided. However, this new state often introduces its own cosmological problems, and is not necessarily theoretically motivated.

In this paper, we show that a promising new state does in fact exist: an uneaten goldstino. This mode arises naturally in the general framework of ref. [10], where multiple sectors separately break SUSY, yielding a corresponding multiplicity of goldstini. In this framework, the gravitino couplings are not modified, but the LOSP can decay faster to an uneaten goldstino, nullifying the usual BBN constraint. Moreover, in the limit where the SUSY breaking sectors are mutually sequestered, the goldstini acquire a mass from SUGRA effects which is exactly twice the gravitino mass. Intriguingly, this factor of 2 is entirely fixed by the symmetries of SUGRA.

As we will see, the scenario outlined above leads to completely consistent cosmologies with a heavy gravitino $m_{3 / 2} \approx O(10-100 \mathrm{GeV})$. This allows one to probe SUGRA via precision studies of charged LOSP decays at the LHC. Furthermore, the LOSP will generically have non-negligible branching fractions into both the gravitino and a goldstino (see figure 1), allowing for a measurement of the relative factor of 2 between their masses. Measuring $M_{\mathrm{Pl}}$ and observing this "smoking gun" factor of 2 would reveal a number of exceedingly deep facts about our universe - not only that SUGRA is correct, but also that SUSY breaking is a generic phenomenon and sequestering is realized in nature. These in 
turn suggest the existence of extra dimensions in which sequestering is naturally realized.

We find it remarkable that consistent cosmological histories happen to favor regions of parameter space in which dramatic LHC signatures are accessible. In fact, by requiring thermal leptogenesis, the gravitino mass must be larger than $\approx 10 \mathrm{GeV}$, with the right abundance for gravitino dark matter obtained for reheating temperatures $T_{R} \approx$ $\left(10^{9}-10^{10}\right) \mathrm{GeV}$. This setup is possible if the goldstino interactions satisfy certain simple conditions, namely that they preserve an $R$ symmetry. More generally, consistent cosmologies are obtained with $T_{R}$ as high as $\approx 10^{7} \mathrm{GeV}$, in which case dark matter is dominantly the goldstino. In both these cases, cosmology prefers the LOSP branching ratios into gravitinos and goldstinos to be not too dissimilar.

The remainder of the paper is organized as follows. We review the collider tests of SUGRA and their tension with BBN in section 2, and show how modified LOSP decays can simply relieve this tension. In section 3, we summarize the framework of multiple SUSY breaking and the important properties of the resulting goldstini [10]. The collider phenomenology of gravitinos/goldstinos is discussed in section 4, and their cosmology is studied in section 5, where the relevant calculation of goldstino relic abundance is summarized in the appendix. The possibility of colored LOSPs is discussed in section 6. Finally, we conclude in section 7 .

\section{$2 \mathrm{BBN}$ and the $\mathrm{LHC}$}

Testing the relationship between the mass and interaction strength of the gravitino requires precision collider measurements which are only feasible if the LOSP is charged. Typically, the LOSP is taken to be a long-lived slepton (most commonly a stau) because this is favored in many SUSY breaking mediation schemes. While other charged states are also possible, we will mostly focus on a slepton LOSP in this paper, leaving a discussion of other possibilities to section 6 .

At the LHC, quasi-stable charged sleptons may be copiously produced at the end of SUSY cascade decays. Most of them will exit the interaction region appearing as "heavy muons." The slepton mass can then be determined from a combination of time-of-flight and momentum information [11-13]. Through ionization energy loss, a fraction of the produced sleptons will also be trapped inside the main detector $[14,15]$ or in a separate stopper detector $[2,16,17]$, where their decays can be precisely studied.

The decay rate of the slepton is measured by observing the lifetime of stopped sleptons. In the conventional SUSY setup, a slepton decays to a lepton and a gravitino, with a width given by

$$
\Gamma_{\tilde{\ell} \rightarrow \ell \tilde{G}} \simeq \frac{m_{\tilde{\ell}}^{5}}{16 \pi F_{\text {tot }}^{2}},
$$

where $F_{\text {tot }}$ is the scale of SUSY breaking, and we have ignored the relatively unimportant phase space factor. From the energy spectrum of the outgoing lepton and the measured slepton mass, one can also determine the gravitino mass, which is fixed by theory to be

$$
m_{3 / 2} \simeq \frac{F_{\mathrm{tot}}}{\sqrt{3} M_{\mathrm{Pl}}} .
$$


The gravitino mass/interaction relation can then be tested [1] by combining the measured values of $\Gamma_{\tilde{\ell} \rightarrow \ell \tilde{G}}$ and $m_{3 / 2}$ to form the Planck scale $M_{\mathrm{Pl}}$

$$
M_{\mathrm{Pl}}^{2} \simeq \frac{m_{\tilde{\ell}}^{5}}{48 \pi \Gamma_{\tilde{\ell} \rightarrow \ell \tilde{G}} m_{3 / 2}^{2}},
$$

and comparing it with the value obtained in long distance measurements of gravity.

However, an indirect measurement of $M_{\mathrm{Pl}}$ is only possible if the mass of the gravitino is sufficiently heavy that it can be experimentally determined. In particular, for a given slepton decay, $m_{3 / 2}$ is reconstructed from the slepton mass and the energy of the outgoing lepton $E_{\ell}$ according to

$$
m_{3 / 2}=\sqrt{m_{\tilde{\ell}}^{2}+m_{\ell}^{2}-2 m_{\tilde{\ell}} E_{\ell}}
$$

Thus, the error in $m_{3 / 2}$ is given by

$$
\frac{\Delta m_{3 / 2}}{m_{3 / 2}} \simeq \frac{m_{\tilde{\ell}}^{2}}{2 m_{3 / 2}^{2}}\left(\Delta_{m} \oplus \Delta_{E}\right),
$$

where $\Delta_{m} \equiv \Delta m_{\tilde{\ell}} / m_{\tilde{\ell}}$ and $\Delta_{E} \equiv \Delta E_{\ell} / E_{\ell}$. With sufficient statistics, we expect that $\Delta m_{\tilde{\ell}}$ and $\Delta_{E}$ can reach the level of $(0.1-1) \%$ at the LHC $[11-13,18,19]$. This implies that the $M_{\mathrm{Pl}}$ measurement is possible for

$$
m_{3 / 2} \gtrsim(0.05-0.2) m_{\tilde{\ell}}
$$

where we have required $\Delta m_{3 / 2} \ll m_{3 / 2}$. Of course, the precise numbers are subject to the level of experimental accuracy which may ultimately be achieved.

In standard SUSY, the mass and interaction strength of the gravitino obey a fixed relation, so any theory with a gravitino heavy enough to satisfy eq. (2.6) will have commensurately long-lived sleptons. As a consequence, this class of theories is in direct tension with BBN. Specifically, sleptons produced in the early universe will decay during or after BBN and potentially alter the abundances of light elements. As seen in ref. [5], the BBN constraint on late-decaying slepton LOSPs implies

$$
m_{3 / 2} \lesssim \begin{cases}0.35 \mathrm{GeV}\left(\frac{m_{\tilde{\ell}}}{100 \mathrm{GeV}}\right)^{2.3} & \text { for } m_{\tilde{\ell}} \lesssim 400 \mathrm{GeV}, \\ 20 \mathrm{GeV}\left(\frac{m_{\tilde{\ell}}}{1 \mathrm{TeV}}\right)^{1.0} & \text { for } m_{\tilde{\ell}} \gtrsim 400 \mathrm{GeV} .\end{cases}
$$

Here a typical primordial slepton yield of $Y_{\tilde{\ell}} \simeq 7 \times 10^{-14}\left(m_{\tilde{\ell}} / 100 \mathrm{GeV}\right)$ was assumed, but the bound depends only weakly on this value. Thus, one sees that the criterion for measuring $M_{\mathrm{Pl}}$ at colliders, eq. (2.6), is in conflict with the BBN bound in conventional SUSY theories. Note that the couplings of the gravitino are completely fixed by $m_{3 / 2}$, so there is no freedom to modify the slepton decay width to the gravitino.

Nevertheless, while the partial width of the slepton into the gravitino is fixed by SUGRA, it is of course possible to change the total width of the slepton. By introducing a new light degree of freedom $\zeta$ and a new decay mode

$$
\tilde{\ell} \rightarrow \ell \zeta
$$


the slepton can decay much more quickly and thus evade constraints from BBN. In fact, a new field $\zeta$ arises quite naturally in the framework of multiple sector SUSY breaking, where $\zeta$ is identified as an uneaten goldstino. We will review this framework in the next section.

In order to test the gravitino mass/interaction relation at the LHC, it is necessary that the slepton has a non-negligible branching fraction to gravitinos. Assuming that there are $O\left(10^{3}-10^{4}\right)$ stopped LOSPs (which correspond to relatively light superpartners with $(100-1000) \mathrm{fb}^{-1}$ of integrated luminosity [2]) we need $\operatorname{Br}_{\tilde{\ell} \rightarrow \ell \tilde{G}} \gtrsim O\left(10^{-4}-10^{-3}\right)$.

In summary, in order to measure $M_{\mathrm{Pl}}$ at colliders and simultaneously evade constraints from BBN, the following conditions must be satisfied:

$$
\begin{aligned}
\Gamma_{\tilde{\ell} \rightarrow \ell \zeta} & \gtrsim\left\{\begin{array}{l}
9.1 \times 10^{-29} \mathrm{GeV}\left(\frac{m_{\tilde{\ell}}}{100 \mathrm{GeV}}\right)^{0.4}\left(m_{\tilde{\ell}} \lesssim 400 \mathrm{GeV}\right), \\
2.8 \times 10^{-27} \mathrm{GeV}\left(\frac{m_{\tilde{\ell}}}{1 \mathrm{TeV}}\right)^{3.0} \quad\left(m_{\tilde{\ell}} \gtrsim 400 \mathrm{GeV}\right),
\end{array}\right. \\
\frac{\Gamma_{\tilde{\ell} \rightarrow \ell \tilde{G}}}{\Gamma_{\tilde{\ell} \rightarrow \ell \zeta}} & \gtrsim O\left(10^{-4}-10^{-3}\right), \\
m_{3 / 2} & \gtrsim(0.05-0.2) m_{\tilde{\ell}},
\end{aligned}
$$

where the first condition has been translated from eq. (2.7) and so has a mild dependence on the primordial LOSP yield $Y_{\tilde{\ell}}$.

As shown in ref. [10] and reviewed below, the mass of an uneaten goldstino is fixed by the symmetries of SUGRA to be $2 m_{3 / 2}$. Consequently, if the gravitino mass is heavy enough to be determined at colliders, then so too is the mass of the goldstino. Thus, we are presented with the intriguing prospect of measuring both decay channels to gravitino and goldstino, as well as the remarkable factor of 2 in the mass relation.

\section{Review of Goldstini framework}

In principle, any light mode $\zeta$ which couples with sufficient strength to the LOSP can nullify BBN constraints. Here we will focus on the framework introduced in ref. [10], where $\zeta$ is an uneaten goldstino which arises in the context of multiple sector SUSY breaking. We find this a particularly attractive possibility both because it is well-motivated from top-down considerations and because it allows for a direct experimental probe of the fundamental properties of spacetime at colliders. In what follows, we briefly review the case of two sectors which independently break SUSY, and refer the interested reader to ref. [10] for a significantly more detailed treatment.

Consider two sectors which separately experience $F$-term SUSY breaking at the scales $F_{1}$ and $F_{2}$, yielding two corresponding goldstini fields, $\eta_{1}$ and $\eta_{2}$. (We take $F_{1}>F_{2}$ without loss of generality.) Because SUSY is a local symmetry, a diagonal combination of these goldstini is eaten by the gravitino via the super-Higgs mechanism, while the remaining orthogonal mode persists as a physical degree of freedom. We can go to the physical mass basis via the transformation

$$
\left(\begin{array}{l}
\eta_{1} \\
\eta_{2}
\end{array}\right)=\left(\begin{array}{cc}
\cos \theta & -\sin \theta \\
\sin \theta & \cos \theta
\end{array}\right)\left(\begin{array}{c}
\eta_{\text {long }} \\
\zeta
\end{array}\right),
$$


where $\tan \theta=F_{2} / F_{1}$. Here $\eta_{\text {long }}$ is the longitudinal mode of the gravitino, while $\zeta$ is the uneaten goldstino which remains in the spectrum. Since the overall scale of SUSY breaking is $F_{\text {tot }}=\sqrt{F_{1}^{2}+F_{2}^{2}}$, the gravitino mass is $m_{3 / 2}=F_{\text {tot }} / \sqrt{3} M_{\mathrm{Pl}}$.

The couplings of each goldstino to chiral and vector superfields of the supersymmetric standard model (SSM) are

$$
\begin{aligned}
& \mathcal{L}_{\phi}=\left(\frac{\tilde{m}_{1}^{2}}{F_{1}} \eta_{1}+\frac{\tilde{m}_{2}^{2}}{F_{2}} \eta_{2}\right) \psi \phi^{\dagger}+\text { h.c. } \\
& \mathcal{L}_{\lambda}=-\frac{i}{\sqrt{2}}\left(\frac{M_{1}}{F_{1}} \eta_{1}+\frac{M_{2}}{F_{2}} \eta_{2}\right) \sigma^{\mu \nu} \lambda F_{\mu \nu}+\text { h.c. }
\end{aligned}
$$

where $\phi, \psi$, and $\lambda$ represent SSM scalars, fermions, and gauginos, respectively. The soft mass terms $\tilde{m}_{1,2}^{2}$ and $M_{1,2}$ are the contributions to the scalar squared masses and gaugino masses from sectors 1 and 2, respectively. We primarily consider a regime in which the SUSY breaking scale of sector 1 is sufficiently larger than that of sector 2 , so that $F_{\text {tot }} \approx$ $F_{1} \gg F_{2}$. In this limit, the couplings are

$$
\begin{aligned}
\mathcal{L}_{\phi} & \approx\left(\frac{\tilde{m}_{1}^{2}+\tilde{m}_{2}^{2}}{F_{\text {tot }}} \eta_{\text {long }}+\frac{\tilde{m}_{2}^{2}}{F_{2}} \zeta\right) \psi \phi^{\dagger}+\text { h.c. } \\
\mathcal{L}_{\lambda} & \approx-\frac{i}{\sqrt{2}}\left(\frac{M_{1}+M_{2}}{F_{\text {tot }}} \eta_{\text {long }}+\frac{M_{2}}{F_{2}} \zeta\right) \sigma^{\mu \nu} \lambda F_{\mu \nu}+\text { h.c. }
\end{aligned}
$$

As long as $\tilde{m}_{2}^{2}$ and $M_{2}$ are not too small, the SSM fields will couple more strongly to the uneaten goldstino $\zeta$ than to the longitudinal mode of the gravitino $\eta_{\text {long }}$, allowing for substantial departures from usual SUGRA signatures.

In ref. [10], it was shown that $\zeta$ (and more generally, any additional uneaten goldstini) acquires a mass

$$
m_{\zeta}=2 m_{3 / 2}+\delta m
$$

where $\delta m$ vanishes in the limit that sectors 1 and 2 are sequestered from each other. We note that the ratio $m_{\zeta} / m_{3 / 2}=2$ is truly a SUGRA prediction, and measuring this ratio would give valuable insight into the structure of spacetime, independent of details of the mechanism of SUSY breaking.

Below we will also consider scenarios in which sectors 1 and 2 both couple to the SSM, in which case the sequestered limit is only an approximation. While direct interactions between sectors 1 and 2 are induced through loops of SSM fields, the resulting $\delta m$ is generally a loop factor or more down in size from SSM soft masses [10] and can be ignored in most of the parameter regions we will be interested.

\section{Gravitino and Goldstini at colliders}

In this section, we will consider the collider phenomenology of LOSP decays to gravitinos and goldstini. For simplicity, we focus on the case of two SUSY breaking sectors, with $F_{1}>F_{2}$. We will be concerned with the regime in which $\tilde{m}_{1}^{2} \lesssim \tilde{m}_{2}^{2}$, so that the SSM fields couple more strongly to the uneaten goldstino $\zeta$ than to the gravitino $\tilde{G}$. The opposite regime has phenomenology which is essentially identical to that of standard SUSY. 


$$
\Gamma_{\tilde{\ell} \rightarrow \ell \tilde{G}} / \Gamma_{\tilde{\ell} \rightarrow \ell \zeta} \text { for } m_{\tilde{\ell}}=100 \mathrm{GeV}
$$
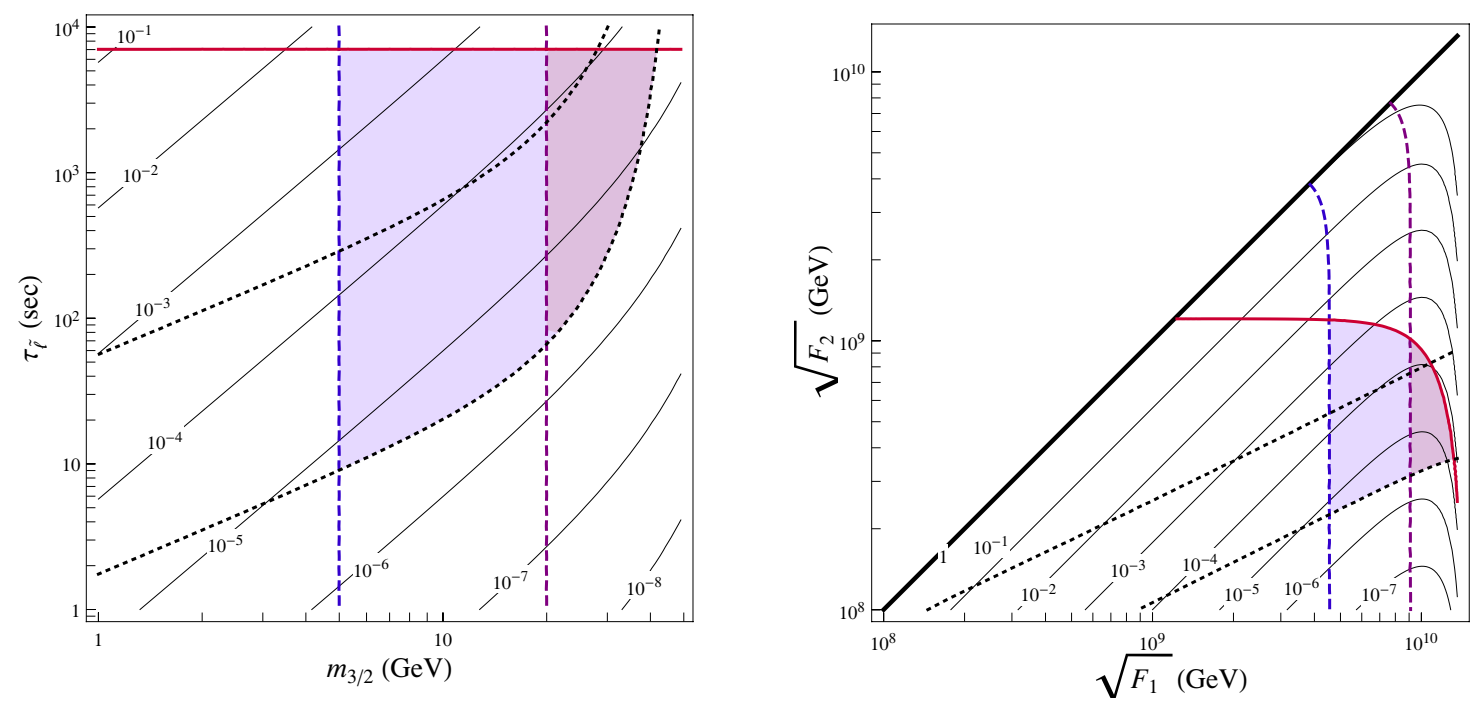

Figure 2. Contours of the branching ratio $\Gamma_{\tilde{\ell} \rightarrow \ell \tilde{G}} / \Gamma_{\tilde{\ell} \rightarrow \ell \zeta}$ (labeled, solid black) together with constraints from cosmology and collider physics for $m_{\tilde{\ell}}=100 \mathrm{GeV}$, shown in the $m_{3 / 2}-\tau_{\tilde{\ell}}$ plane (left) and in the $\sqrt{F_{1}}-\sqrt{F_{2}}$ plane (right). The BBN bound excludes the parameter regions above the solid red lines, while goldstino overproduction from SSM sfermion decays excludes the regions below the dotted lines (the two dotted lines in each plot correspond to $r \equiv m_{\tilde{Q}} / m_{\tilde{L}}=3$ (lower) and 10 (upper); see section 5.1). Demanding that the gravitino is heavy enough to be measured at colliders places a lower bound on the gravitino mass depending on experimental resolutions, restricting to the regions right of the vertical dashed lines (blue for $m_{3 / 2}>0.05 m_{\tilde{\ell}}$ and purple for $m_{3 / 2}>0.2 m_{\tilde{\ell}}$ ). The parameter regions consistent with all the constraints are shaded. To read off analogous bounds on the conventional SUSY setup, simply restrict to the line $F_{1}=F_{2} \equiv F$.

Consider the limiting case $\tilde{m}_{1}^{2} \ll \tilde{m}_{2}^{2}$; extensions to more general cases are straightforward. In this limit, the partial widths of the LOSP into the gravitino and the goldstino take particularly simple forms. As in section 2, we assume a charged slepton LOSP, so

$$
\begin{aligned}
& \Gamma_{\tilde{\ell} \rightarrow \ell \tilde{G}} \simeq \frac{m_{\tilde{\ell}}^{5}}{16 \pi F_{\mathrm{tot}}^{2}}, \\
& \Gamma_{\tilde{\ell} \rightarrow \ell \zeta} \simeq \frac{m_{\tilde{\ell}}^{5}}{16 \pi F_{2}^{2}},
\end{aligned}
$$

where we have dropped phase space factors for simplicity. Using the formulas from section 2, let us now determine the region of parameter space in which the BBN bound is satisfied and the gravitino (and goldstino) masses can be measured at the LHC. As discussed in ref. [10], the decay rate of goldstinos to gravitinos is cosmological and therefore irrelevant for our discussions here.

The regions of parameter space which satisfy eqs. (2.9) and (2.11) are shown in figure 2 (figure 3) for $m_{\tilde{\ell}}=100 \mathrm{GeV}(300 \mathrm{GeV})$. The left and right panels depict these allowed regions in the $m_{3 / 2}-\tau_{\tilde{\ell}}$ and $F_{1}-F_{2}$ planes, respectively, where $\tau_{\tilde{\ell}}$ is the LOSP lifetime. In producing these plots, we have included the phase space factors and higher order terms 

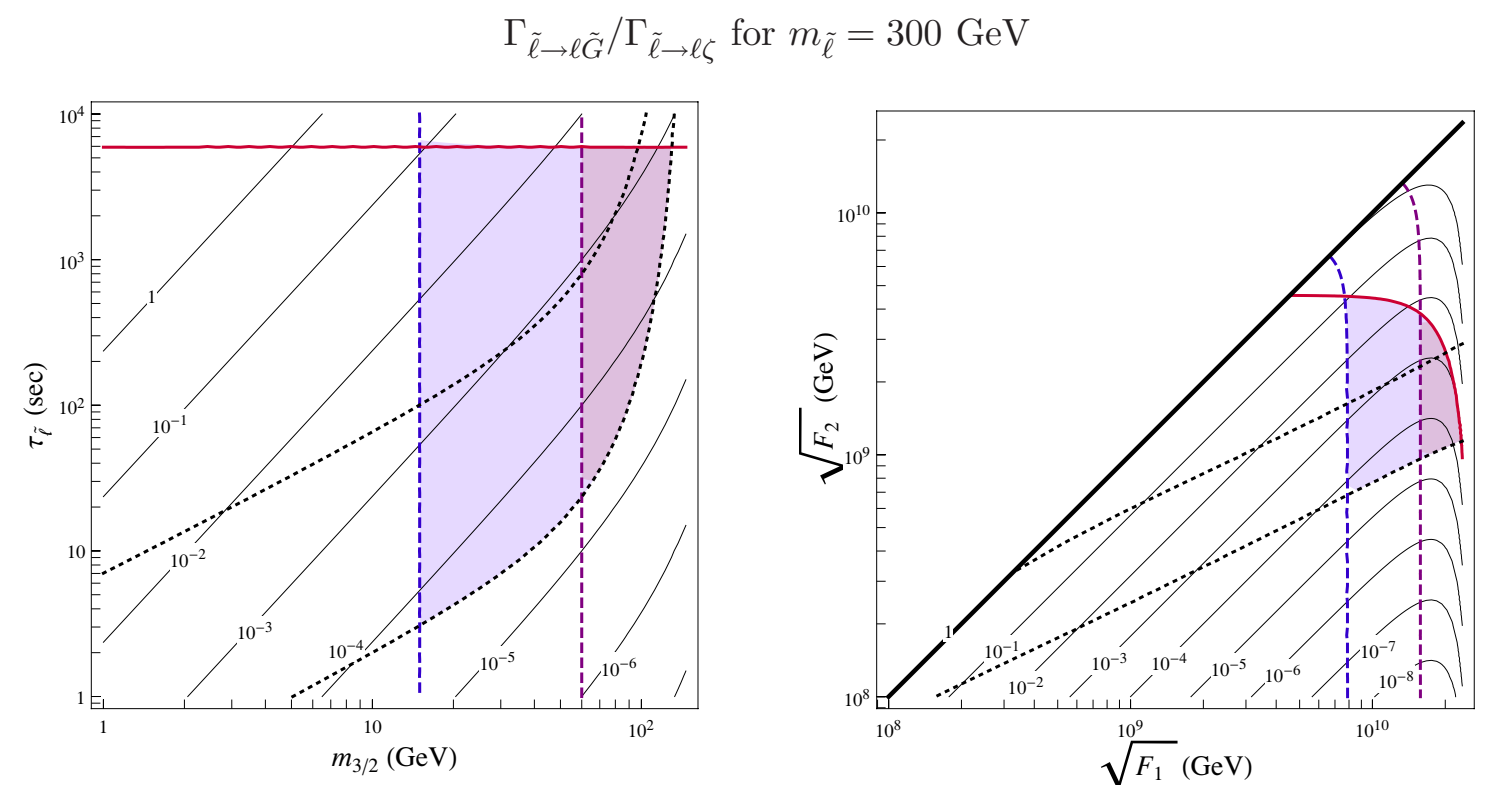

Figure 3. The same as figure 2 but for $m_{\tilde{\ell}}=300 \mathrm{GeV}$.

in $F_{2} / F_{1}$ which are omitted in eqs. (4.1) and (4.2). In each plot, the region below the solid line is allowed by $\mathrm{BBN}$, while the regions right of the vertical, dashed lines satisfy eq. (2.11) (with the two lines corresponding to $m_{3 / 2} / m_{\tilde{\ell}}=0.05$ and 0.2 ). The two dotted lines represent the cosmological bound discussed in the next section. The labeled contours denote the branching ratio of $\Gamma_{\tilde{\ell} \rightarrow \ell \tilde{G}} / \Gamma_{\tilde{\ell} \rightarrow \ell \zeta}$, which must be sufficiently large if we are to be able to see LOSP decays to both gravitinos and goldstinos.

A number of important facts are evident from these plots. In particular, we can immediately see the direct conflict between collider signatures and BBN in conventional SUSY by considering the plot on the right panel and restricting to the diagonal line $F_{1}=$ $F_{2} \equiv F$. As expected, along this line, there is no region of parameter space in which the gravitino is heavy enough to be measured at colliders and also simultaneously consistent with BBN constraints. However, moving down and to the right (regions with $F_{2}<F_{1}$ ), we find that a viable parameter space does open up. Nonetheless, even this region of parameter space is limited by cosmological considerations, as we will see in the next section. The viable parameter space is thus a finite region in the $F_{1}-F_{2}$ (and $m_{3 / 2}-\tau_{\tilde{\ell}}$ ) plane, so that the branching ratio $\Gamma_{\tilde{\ell} \rightarrow \ell \tilde{G}} / \Gamma_{\tilde{\ell} \rightarrow \ell \zeta}$ has a lower bound, which is of $O\left(10^{-5}\right)$ or so. This value is not far from the limit of LHC observability, given in eq. (2.10). It is also interesting that resulting LOSP lifetimes, $\tau_{\tilde{\ell}} \approx O\left(1-10^{4} \mathrm{sec}\right)$, are within the range in which stopped LOSP decays may be observed in the main detector $[14,15]$.

In order to measure the Planck scale from decays of long-lived charged LOSPs, we form a combination of the LOSP mass and lifetime and the mass of the invisible LOSP decay product; see eq. (2.3). If the decay product is indeed the gravitino, this should reproduce the true Planck scale, $M_{\mathrm{Pl}}$. In our case, however, the LOSP decays mainly into $\zeta$, so that the measured "Planck scale", " $M_{\mathrm{Pl}}$ ", will deviate from $M_{\mathrm{Pl}}$ by

$$
" M_{\mathrm{Pl}} " 2 \equiv \frac{m_{\tilde{\ell}}^{5}}{48 \pi \Gamma_{\tilde{\ell} \rightarrow \ell \zeta} m_{\zeta}^{2}}=M_{\mathrm{Pl}}^{2} \frac{\Gamma_{\tilde{\ell} \rightarrow \ell \tilde{G}}}{\Gamma_{\tilde{\ell} \rightarrow \ell \zeta}} \frac{m_{\tilde{G}}^{2}}{m_{\zeta}^{2}},
$$


where again we have dropped phase space factors for the sake of clarity. Consequently, we expect to measure a value for " $M_{\mathrm{Pl}}$ " which is slightly (one or two orders of magnitude) lower than $M_{\mathrm{Pl}}$. Interestingly, the measured value of " $M_{\mathrm{Pl}}$ " can be used to precisely fix the branching ratio of the LOSP to the gravitino

$$
\frac{\Gamma_{\tilde{\ell} \rightarrow \ell \tilde{G}}}{\Gamma_{\tilde{\ell} \rightarrow \ell \zeta}} \simeq 4\left(\frac{" M_{\mathrm{Pl}} "}{M_{\mathrm{Pl}}}\right)^{2},
$$

where $m_{\zeta} \simeq 2 m_{3 / 2}$ has been used. Thus, by measuring " $M_{\mathrm{Pl}}$ " we know how many stopped LOSPs are necessary to observe the second peak in $E_{\ell}$ which corresponds to the gravitino. The Planck scale constructed from this second peak should then reproduce the value obtained by macroscopic measurements, $M_{\mathrm{Pl}}$.

\section{Viable cosmologies}

It is reasonable to ask to what extent the collider signature discussed in the previous section is consistent with cosmology. For example, if the reheating temperature, $T_{R}$, is smaller than the SSM superparticle mass scale, then the only constraints on the masses and couplings of gravitinos and goldstinos come from BBN. If $T_{R}$ is smaller than the LOSP freezeout temperature, then even the constraint from BBN disappears.

However, most standard cosmologies require a significantly higher reheating temperature, in which case one must evade constraints from the overproduction of gravitinos and goldstinos as well as the BBN bound. We discuss these constraints in section 5.1, and present a number of consistent cosmological scenarios with high $T_{R}$ in the subsequent subsections. In each setup, either the goldstino or the gravitino could comprise the dark matter of the universe. Throughout this section, we assume the absence of significant entropy production below $T_{R}$, which is indeed the case for most standard cosmologies.

\subsection{Reheating bounds on Goldstini couplings}

Avoiding goldstino/gravitino overproduction in the early universe may provide bounds on $T_{R}$ and their interactions. In the case of the gravitino, this sets a robust upper bound on $T_{R}$ as a function of $m_{3 / 2}[7,8]$. In contrast, the bounds from goldstino overproduction depend on the goldstino interactions with the SSM fields - unlike the gravitino, the goldstino can have couplings to the SSM which are not universal.

Suppose that sector 2 provides soft mass contributions to all the SSM superparticles. In this case, the goldstino couples to SSM fields (almost) universally. Since its couplings are larger than those of the gravitino by a factor of $F_{\text {tot }} / F_{2}$, the overproduction bound is correspondingly more stringent. In particular, the standard gravitino overproduction bound can be straightforwardly translated into a bound on goldstino overproduction via ref. [10]

$$
T_{R}^{\max } \approx 10^{5} \mathrm{GeV}\left(\frac{10 \mathrm{GeV}}{m_{\zeta}}\right)\left(\frac{\sqrt{F_{2}}}{10^{9} \mathrm{GeV}}\right)^{4}
$$



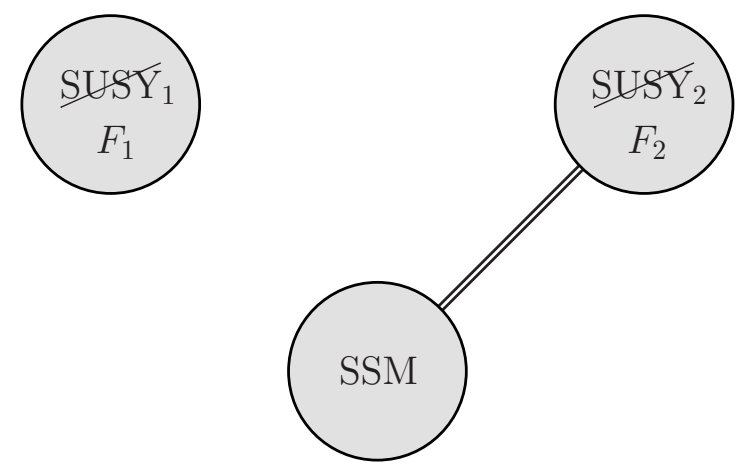

Figure 4. Minimal setup in which a standard SUSY breaking scheme (SSM + sector 2) is augmented by an additional sequestered sector which happens to break SUSY at some higher scale (sector 1).

for $T_{R}^{\max }$ larger than the SSM superparticle masses. As in the case of the gravitino, the production of goldstinos in this case is dominated at high temperatures by processes involving gauge-gaugino-goldstino vertices. This is because these vertices are dimension 5 operators.

As shown in section 5.3, however, the gauge-gaugino-goldstino interactions can be effectively removed using an $R$ symmetry. In this case, the bound from cosmological goldstino overproduction is far milder, since the goldstino couples very weakly to the gauginos. Instead, the leading overproduction bound arises from processes involving the scalarfermion-goldstino couplings, which are dimension 4 interactions. Since the strength of these interactions do not grow with temperature, the production of goldstinos through decays and scatterings involving SSM states is dominated by the infrared. Thus, the primary constraint from goldstino overproduction is a $T_{R}$-independent bound on the scalar-fermiongoldstino couplings.

As discussed in the appendix, the leading contribution to goldstino production through scalar-fermion-goldstino vertices comes from superparticle decays. Since the relevant amplitudes scale with $1 / F_{2}$, this sets a lower bound on $m_{\zeta} / F_{2}^{2} \propto F_{\text {tot }} / F_{2}^{2}$. The precise bound depends on the spectrum of superparticles (since the goldstino couplings depend on the superparticle masses), and for concreteness, we consider $m_{\tilde{Q}}=r m_{\tilde{L}}$ with $r=3$ and 10, where $m_{\tilde{Q}, \tilde{L}}$ are the squark and slepton masses taken, for simplicity, to be universal at the weak scale. These bounds are depicted in figures 2 and 3 as dotted lines, and given roughly by

$$
\frac{F_{2}^{2}}{F_{\text {tot }}} \gtrsim 10^{14} \mathrm{GeV}^{2}\left(\frac{m_{\tilde{Q}}}{300 \mathrm{GeV}}\right)^{3}
$$

\subsection{The minimal Goldstini scenario}

In the minimal goldstini scenario, the SSM couples directly to sector 2 but not to sector 1, so that $\tilde{m}_{1}^{2}$ and $M_{1}$ both vanish (see figure 4 ). This corresponds to a setup in which a standard SUSY breaking scheme (i.e. SSM + sector 2) is augmented by a single sequestered sector which happens to break SUSY at some higher scale (i.e. sector 1). Such constructions are expected to arise rather naturally from ultraviolet theories. 


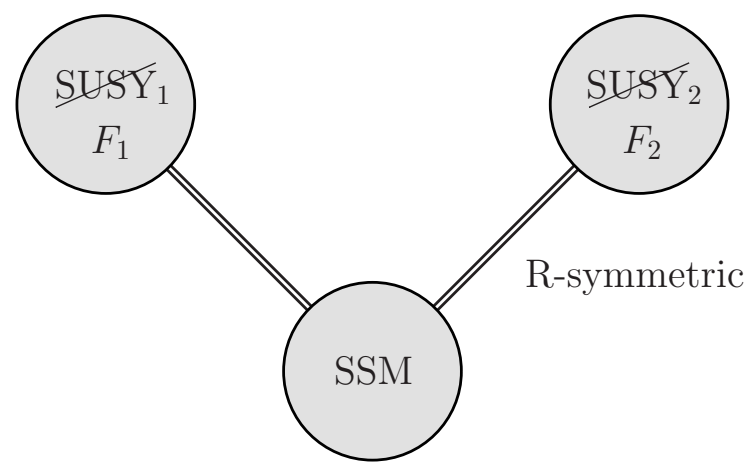

Figure 5. Setup in which both sector 1 and sector 2 couple directly to the SSM. By construction, couplings between sector 2 and the SSM are $R$ symmetric, so gaugino masses arise solely from sector 1. For the same reason, the goldstino has suppressed couplings to gauginos.

Since this minimal setup contains gauge-gaugino-goldstino vertices, the bound on the reheating temperature from eq. (5.1) applies. Consequently, the scalar-fermion-goldstino couplings are important only when $T_{R}^{\max }$ is close to the SSM superparticle masses, yielding $O(1)$ corrections to eq. (5.1). For low enough $T_{R}$, only the $T_{R}$-independent bound from eq. (5.2) is relevant, as depicted in figures 2 and 3.

When the bound of eq. (5.1) is saturated, $T_{R} \approx T_{R}^{\max }$, the goldstino comprises all of the dark matter in the universe. The production is dominated by the ultraviolet and is thus sensitive to the value of $T_{R}$. Comparing eq. (5.1) to the allowed regions in figures 2 and 3 , we find that $T_{R}$ as high as $\approx 10^{7} \mathrm{GeV}$ (corresponding to $m_{\zeta} \simeq$ few $\times 10 \mathrm{GeV}$, $F_{2} \simeq$ few $\times 10^{9} \mathrm{GeV}$ ) can be consistent with the BBN bound and the collider measurement of $M_{\mathrm{Pl}}$. Such a high reheating temperature allows for high temperature mechanisms for baryogenesis that would otherwise not work with $T_{R} \lesssim m_{\mathrm{LOSP}} / 20$, although it is still too low for thermal leptogenesis. Note that the gravitino abundance is small, $\Omega_{3 / 2} \approx$ $\left(F_{2} / F_{\text {tot }}\right)^{2} \Omega_{\zeta}$, and that the goldstino energy density coming from late decays of the LOSP after LOSP freezeout is also typically subdominant.

\subsection{SUSY breaking with $R$ symmetries}

Copious production of goldstinos at very high temperatures is not inevitable. In particular, since the bound on the reheating temperature eq. (5.1) is wholly determined by the goldstino couplings to the gauginos, it can be evaded by imposing an $R$ symmetry. This allows for an alternative cosmological scenario with non-thermal gravitino dark matter.

Consider the setup depicted in figure 5, where sector 2 preserves an $R$ symmetry. In this case, the sfermion masses receive a contribution from sector $2, \tilde{m}_{2}^{2} \neq 0$, but not the gaugino masses, $M_{2}=0$. Sector 1 , which does not preserve an $R$ symmetry, generates both $\tilde{m}_{1}^{2}$ and $M_{1}$. For simplicity, we consider that the resulting sfermion and gaugino masses are of the same order, which can easily happen if $\tilde{m}_{2}^{2}$ is not much larger than $M_{1}^{2}$. Our analysis below assumes that the dominant contribution to the sfermion masses comes from sector 2, although the existence of a comparable contribution from sector 1 does not change our essential conclusions. 
In this $R$-symmetric setup, the bound from goldstino overproduction is quite mild because the goldstino couples very weakly to the gauginos. The only relevant interactions are the scalar-fermion-goldstino couplings, so we need only consider the $T_{R}$-independent bound from eq. (5.2) which are shown in figures 2 and 3. It is interesting that this cosmological bound leads to a lower limit on the branching fraction $\Gamma_{\tilde{\ell} \rightarrow \ell \tilde{G}} / \Gamma_{\tilde{\ell} \rightarrow \ell \zeta} \gtrsim O\left(10^{-5}\right)$, which favors the possibility of observing both $\tilde{G}$ and $\zeta$ at the LHC.

Since the coupling strength of the gauginos to the goldstino is a factor of $F_{2} / F_{1}$ weaker than to the gravitino in the present setup, the constraint from goldstino overproduction through these couplings is weaker than that from gravitino overproduction. From figures 2 and 3, parameter regions we are interested in are roughly $F_{2} \sim 10^{9} \mathrm{GeV}$ and $F_{\text {tot }} \approx F_{1} \sim$ $10^{10} \mathrm{GeV}$. The bound on $T_{R}$ from gravitino overproduction in these parameter regions is rather weak $[7,8]$

$$
T_{R}^{\max } \approx O\left(10^{8}-10^{10} \mathrm{GeV}\right),
$$

so that it can even be compatible with thermal leptogenesis, which typically requires $T_{R} \gtrsim$ $10^{9} \mathrm{GeV}$. If the bound of eq. (5.3) is saturated, we have gravitino dark matter.

Note that in conventional SUSY breaking scenarios, gravitino dark matter with a high reheating temperature such as in eq. (5.3) is not possible, due to stringent constraints from $\mathrm{BBN}$. In our case, however, the LOSP decays to the goldstino faster than to the gravitino as long as $\tilde{m}_{2}^{2} / F_{2}$ is sufficiently large. This allows us to evade the BBN bound consistently with gravitino dark matter and thermal leptogenesis.

\subsection{Late decay case}

So far, we have assumed that the relic density of goldstinos arising from late LOSP decays is small. This is true in most of the natural parameter regions, but in certain corners of parameter space, goldstinos from late LOSP decays may saturate the observed dark matter abundance.

Suppose that the slepton freezeout abundance is completely controlled by annihilation into gauge bosons (which will be the case if the neutralinos and heavy Higgs bosons are sufficiently heavier than the slepton). In this case, the yield of the (mostly right-handed) slepton before its decay is given by $Y_{\tilde{\ell}} \simeq 2 \times 10^{-13}\left(m_{\tilde{\ell}} / 100 \mathrm{GeV}\right)$ [20]. This leads to the goldstino relic abundance

$$
\Omega_{\zeta} \simeq 0.2\left(\frac{m_{\zeta}}{200 \mathrm{GeV}}\right)\left(\frac{m_{\tilde{\ell}}}{1 \mathrm{TeV}}\right)
$$

so that if the slepton is very heavy, dark matter goldstinos may mostly come from late slepton decays. Such heavy sleptons, however, may be problematic for LHC measurements.

\section{Other LOSPs}

In the previous discussion, we have considered the case where the LOSP is a (mostly right-handed) charged slepton. In this section, we briefly discuss other possibilities. 
To achieve the signatures discussed in this paper, there must be a quasi-stable charged state which is stopped either in the main detector or in a stopper detector. This immediately eliminates the possibility of a (mostly) bino LOSP. Similarly, wino, Higgsino or left-handed slepton LOSPs typically do not lead to the relevant signatures, since a mass splitting between the charged and neutral components (induced by radiative corrections, tree-level mixings, or the $D$-term effect) are so large that the charged component decays with lifetime shorter than $\approx 10^{-6}$ sec (see however [21]). With the constraint from overproduction in section 5.1, the branching fraction of a charged component to the goldstino is then tiny, $\lesssim 10^{-6}$.

This leaves only the possibility of a gluino or squark LOSP. In the early universe these states freeze out at a temperature of $O\left(0.1 m_{\mathrm{LOSP}}\right)$, with an abundance determined by perturbative strong interaction processes, $Y_{\mathrm{LOSP}, \text { pert }} \approx 10^{-13}\left(m_{\mathrm{LOSP}} / 1 \mathrm{TeV}\right)$. For the gluino $\tilde{g}$, this abundance will be reduced significantly by nonperturbative annihilations occurring after the QCD phase transition, $Y_{\tilde{g}} \approx 10^{-20}\left(m_{\tilde{g}} / 1 \mathrm{TeV}\right)^{1 / 2}[22]$. On the other hand, for squarks $\tilde{q}$, nonperturbative processes lead to a significant fraction of $\tilde{q} \tilde{q} \tilde{q}$ bound states, which are not subject to enhanced annihilations. Therefore, the squark abundance may not be much reduced from the perturbative value, $Y_{\tilde{q}} \approx O\left(10^{-14}-10^{-13}\right)\left(m_{\tilde{q}} / 1 \mathrm{TeV}\right)$.

With the relic abundance given above, the gluino LOSP does not suffer from the BBN constraint. ${ }^{1}$ On the other hand, squark LOSPs are subject to the BBN constraint coming from hadronic energy injections; conservatively it is $\tau_{\tilde{q}} \lesssim 100 \mathrm{sec}$ [23]. For a fixed LOSP mass, the constraint from goldstino overproduction can be weaker for gluino/squark LOSPs than for slepton LOSPs, since the masses of colored superparticles, which mainly control the goldstino abundance, can be smaller. A conservative constraint is given by eq. (5.2) with $m_{\tilde{Q}}$ replaced by $m_{\text {LOSP }}$, which corresponds to taking $r \simeq 1$.

Gluino/squark LOSPs can be produced at the LHC either directly or through decays of heavier superparticles. After being produced, they hadronize by picking up a gluon $g$ or up/down quarks $q=u, d$. For the gluino, the relevant bound states are $\tilde{g} g, \tilde{g} \bar{q} q$, and $\tilde{g} q q q$. While the precise spectrum of bound states is not obvious, a fraction of gluinos is stopped in the detector under reasonable assumptions [24], allowing for gluino decay measurements (assuming that tracks can be reconstructed despite charge oscillation). The mass of the gluino can also be measured using charged gluino bound states traversing the muon system. The measurement of the Planck scale will thus be feasible for the gluino LOSP. The situation for squark LOSPs is similar, where the relevant bound states are $\tilde{q} \bar{q}$ and $\tilde{q} q q$.

The visible decay products of gluino/squark LOSPs are jets, with an energy resolution expected to be $\Delta_{E} \approx O(1 \%)$. Therefore, to be able to perform the measurements discussed in this paper, the masses of the gravitino and goldstino must be larger than $\approx O(0.1) m_{\mathrm{LOSP}}$; see eq. (2.5).

In summary, the parameter regions in which the goldstino/gravitino collider signals are obtained consistently with high reheating temperatures (i.e. satisfying both the BBN

\footnotetext{
${ }^{1}$ This implies that if the gluino is the LOSP, the collider measurement of $M_{\mathrm{Pl}}$ can be consistent with the BBN bound even in the conventional SUSY framework. The measurement of gluino decays will be discussed below.
} 
and overproduction constraints) are

$$
\tau_{\tilde{g}} \gtrsim \tau_{\min }, \quad m_{3 / 2} \gtrsim O(0.1) m_{\mathrm{LOSP}}
$$

for a gluino LOSP, and

$$
\tau_{\min } \lesssim \tau_{\tilde{q}} \lesssim 100 \mathrm{sec}, \quad m_{3 / 2} \gtrsim O(0.1) m_{\mathrm{LOSP}},
$$

for a squark LOSP. Here,

$$
\tau_{\min }=0.2 \sec \left(\frac{300 \mathrm{GeV}}{m_{\mathrm{LOSP}}}\right)\left(\frac{m_{3 / 2} / m_{\mathrm{LOSP}}}{0.1}\right)
$$

is obtained by translating eq. (5.2) into a bound on LOSP lifetimes ignoring the phase space factor, which, however, would become important when $m_{\zeta} \approx m_{\mathrm{LOSP}}$.

\section{Conclusions}

The LHC may offer an unprecedented opportunity to probe the fundamental structure of spacetime at colliders. In particular, if the LOSP is charged, then precision measurements of its decays to the gravitino could provide a genuine collider measurement of $M_{\mathrm{Pl}}$ and a dramatic confirmation of SUGRA. Unfortunately, this decay process is directly constrained by BBN in the early universe. Thus, there must be some modification of the conventional SUSY framework to allow for high reheat temperatures $T_{R} \gtrsim \mathrm{TeV}$ to be consistent with collider probes of SUGRA.

In this paper, we have shown that the goldstini framework introduced in ref. [10] provides precisely such a modification. Multiple sources of SUSY breaking yield a corresponding multiplicity of goldstini which can easily couple more strongly to the SSM than the gravitino. Thus, the LOSP decays to goldstini fast enough to avoid the BBN bound, while the gravitino mass can still be measured in colliders via the LOSP decay to the gravitino. In fact, the regions in parameter space where this occurs are favored by cosmology.

Intriguingly, within this setup colliders will first measure the LOSP decay to the goldstino. Initially, this will almost certainly be interpreted as a LOSP decay to a gravitino, which will in turn result in a mismeasurement of $M_{\mathrm{Pl}}$ (one or two orders of magnitude below the value obtained from long-distance gravity). As we have shown, the degree of the discrepancy actually fixes the LOSP branching ratio into the gravitino, and hence the amount of integrated luminosity needed to discover the gravitino. Once this target luminosity is reached, our framework can be tested unambiguously. In particular, one may measure the masses of both the gravitino and goldstino, and if these satisfy $m_{\zeta}=2 m_{3 / 2}$ as predicted in ref. [10], then this would provide a smoking gun signature of the goldstini setup. Specifically, we would learn not only that SUGRA is a symmetry of nature, but also that SUSY is broken multiple times and that sequestering is a real phenomenon. This would in turn suggest the existence of compact extra dimensions in which sequestering naturally emerges.

The scenarios described here are consistent with standard cosmology with high reheating temperatures. In particular, if the sector giving the goldstino preserves an $R$ symmetry, 
then the bound from goldstino overproduction does not lead to an extra constraint on $T_{R}$ beyond that from gravitino overproduction. This allows for thermal leptogenesis with LSP (gravitino) dark matter, which is not possible in the standard SUSY framework with $R$-parity.

Note added: related work [26] discussing a similar mechanism for evading BBN constraints appeared concurrently with this paper.

\section{Acknowledgments}

The work of C.C., J.M., and Y.N. was supported in part by the Director, Office of Science, Office of High Energy and Nuclear Physics, of the US Department of Energy under Contract DE-AC02-05CH11231, and in part by the National Science Foundation under grants PHY0555661 and PHY-0855653. J.T. is supported by the U.S. Department of Energy under cooperative research agreement DE-FG0205ER41360.

\section{A Infrared-dominated Goldstino production}

The late-time goldstino yield can be computed with a standard Boltzmann equation calculation. The yield is defined as $Y_{\zeta} \equiv n_{\zeta} / s$, where $n_{\zeta}$ is the goldstino number density and $s$ is the total entropy density, and is constant once goldstino production is completed. There are three potentially relevant goldstino production mechanisms: superparticle decays and $2 \rightarrow 2$ scattering processes in the early thermal bath, and late decays of relic LOSPs after LOSP freezeout. If the goldstino does not couple to gauge multiplets, as occurs in the $R$-symmetric setup described in section 5.3, then the goldstino production is dominated by scalar decays at $T \sim \tilde{m}$, and is insensitive to $T_{R}$. This is in contrast to the standard gravitino production calculation [7], where the goldstino abundance grows linearly with $T_{R}$.

Here, we briefly describe the calculation of the contribution from superparticle decays in this scenario. The contribution from $2 \rightarrow 2$ scattering can be calculated in a similar (but more involved) manner, but we find it to be subdominant and omit it from our analysis. The contribution from late LOSP decays can be taken directly from the LOSP freezeout abundance used to determine the BBN bound [5]. For a slepton LOSP, for example, $Y_{\zeta}^{(\text {LOSP-decay })}=7 \times 10^{-14}\left(m_{\tilde{\ell}} / 100 \mathrm{GeV}\right)$, which is not significant unless $m_{\tilde{\ell}} \gtrsim 700 \mathrm{GeV}$.

The goldstino yield from decays in the thermal bath is found by solving the Boltzmann equation:

$$
\dot{n}_{\zeta}+3 H n_{\zeta}=\sum_{i} n_{i}\left\langle\frac{1}{\gamma}\right\rangle_{i} \Gamma_{i \rightarrow \zeta}
$$

where dots indicate derivatives with respect to time, the sum is over unstable species, $n_{i}$ and $\Gamma_{i \rightarrow \zeta}$ are their number densities and decay rates to goldstinos, and $\langle 1 / \gamma\rangle$ is the thermally averaged relativistic time-dilation factor to account for out-of-rest-frame decay rates. Using the fact that the entropy per comoving volume is constant, we have the relations

$$
\dot{Y}_{\zeta}=\frac{1}{s}\left(\dot{n}_{\zeta}+3 H n_{\zeta}\right)
$$


and

$$
\frac{d t}{d T}=-\frac{1}{H T}\left(1+\frac{1}{3} \frac{d \log g_{S}(T)}{d \log T}\right)
$$

where $T$ is temperature and $g_{S}(T)$ is the effective number of relativistic species. The goldstino yield is thus

$$
Y_{\zeta}=\int_{T_{R}}^{0} d T \frac{d t}{d T} \dot{Y}_{\zeta}
$$

For concreteness, we assume the simple spectrum $m=m_{\text {LOSP }}$ for non-colored particles and $m=r m_{\text {LOSP }}$ for colored particles, with $r$ a free parameter. Squark decays dominate the production process, with a decay width

$$
\Gamma_{\tilde{Q} \rightarrow Q \zeta} \simeq \frac{1}{16 \pi} \frac{m_{\tilde{Q}}^{5}}{F_{2}^{2}} .
$$

Parametrically, for decays

$$
\dot{Y}_{\zeta}^{\text {decay }} \simeq \frac{m_{\tilde{Q}}^{5}}{F_{2}^{2}} \theta\left(T-m_{\tilde{Q}}\right), \quad \frac{d t}{d T} \sim-\frac{M_{\mathrm{Pl}}}{T^{3}} .
$$

Solving the Boltzmann equations numerically, keeping the full temperature dependence, we find:

$$
Y_{\zeta}^{\text {decay }} \approx 0.0013 \frac{M_{\mathrm{Pl}} r^{3} m_{\mathrm{LOSP}}^{3}}{F_{2}^{2}} .
$$

Here, we show only the leading order dependence on $F_{1}, F_{2}$ and $r$, but we keep the full dependence in figures 2 and 3 . The goldstino overabundance bound is set by requiring $m_{\zeta} Y_{\zeta}<3.8 \times 10^{-10} \mathrm{GeV}[25]$, so that the goldstino abundance is not in conflict with the observed dark matter density.

Open Access. This article is distributed under the terms of the Creative Commons Attribution Noncommercial License which permits any noncommercial use, distribution, and reproduction in any medium, provided the original author(s) and source are credited.

\section{References}

[1] W. Buchmüller, K. Hamaguchi, M. Ratz and T. Yanagida, Supergravity at colliders, Phys. Lett. B 588 (2004) 90 [hep-ph/0402179] [SPIRES].

[2] K. Hamaguchi, M.M. Nojiri and A. de Roeck, Prospects to study a long-lived charged next lightest supersymmetric particle at the LHC, JHEP 03 (2007) 046 [hep-ph/0612060] [SPIRES].

[3] M.Y. Khlopov and A.D. Linde, Is it easy to save the gravitino?, Phys. Lett. B 138 (1984) 265 [SPIRES].

[4] J.R. Ellis, J.E. Kim and D.V. Nanopoulos, Cosmological gravitino regeneration and decay, Phys. Lett. B 145 (1984) 181 [SPIRES].

[5] M. Kawasaki, K. Kohri, T. Moroi and A. Yotsuyanagi, Big-Bang nucleosynthesis and gravitino, Phys. Rev. D 78 (2008) 065011 [arXiv:0804.3745] [SPIRES]. 
[6] M. Fukugita and T. Yanagida, Baryogenesis without grand unification, Phys. Lett. B 174 (1986) 45 [SPIRES].

[7] T. Moroi, H. Murayama and M. Yamaguchi, Cosmological constraints on the light stable gravitino, Phys. Lett. B 303 (1993) 289 [SPIRES].

[8] V.S. Rychkov and A. Strumia, Thermal production of gravitinos, Phys. Rev. D 75 (2007) 075011 [hep-ph/0701104] [SPIRES].

[9] M. Viel, J. Lesgourgues, M.G. Haehnelt, S. Matarrese and A. Riotto, Constraining warm dark matter candidates including sterile neutrinos and light gravitinos with WMAP and the

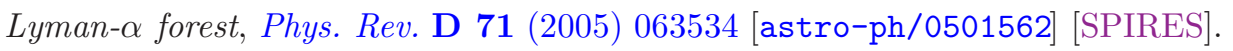

[10] C. Cheung, Y. Nomura and J. Thaler, Goldstini, JHEP 03 (2010) 073 [arXiv:1002.1967] [SPIRES].

[11] I. Hinchliffe and F.E. Paige, Measurements in gauge mediated SUSY breaking models at LHC, Phys. Rev. D 60 (1999) 095002 [hep-ph/9812233] [SPIRES].

[12] S. Ambrosanio, B. Mele, S. Petrarca, G. Polesello and A. Rimoldi, Measuring the SUSY breaking scale at the LHC in the slepton NLSP scenario of GMSB models, JHEP 01 (2001) 014 [hep-ph/0010081] [SPIRES].

[13] J.R. Ellis, A.R. Raklev and O.K. Oye, Gravitino dark matter scenarios with massive metastable charged sparticles at the LHC, JHEP 10 (2006) 061 [hep-ph/0607261] [SPIRES].

[14] S. Asai, K. Hamaguchi and S. Shirai, Stop and decay of long-lived charged massive particles at the LHC detectors, Phys. Rev. Lett. 103 (2009) 141803 [arXiv:0902.3754] [SPIRES].

[15] CMS collaboration, Searching for stopping gluinos during beam-off periods at CMS, http://cms-physics.web.cern.ch/cms-physics/public/EXO-09-001-pas.pdf.

[16] K. Hamaguchi, Y. Kuno, T. Nakaya and M.M. Nojiri, A study of late decaying charged particles at future colliders, Phys. Rev. D 70 (2004) 115007 [hep-ph/0409248] [SPIRES].

[17] J.L. Feng and B.T. Smith, Slepton trapping at the Large Hadron and International Linear Colliders, Phys. Rev. D 71 (2005) 015004 [Erratum ibid. D 71 (2005) 0109904] [hep-ph/0409278] [SPIRES].

[18] ATLAS collaboration, ATLAS physics TDR, http://atlas.web.cern.ch/Atlas/GROUPS/PHYSICS/TDR/TDR.html.

[19] CMS collaboration, CMS Physics TDR, http://cmsdoc.cern.ch/cms/cpt/tdr/index.html.

[20] C.F. Berger, L. Covi, S. Kraml and F. Palorini, The number density of a charged relic, JCAP 10 (2008) 005 [arXiv:0807.0211] [SPIRES].

[21] G.D. Kribs, A. Martin and T.S. Roy, Supersymmetry with a chargino NLSP and gravitino LSP, JHEP 01 (2009) 023 [arXiv:0807.4936] [SPIRES].

[22] J. Kang, M.A. Luty and S. Nasri, The relic abundance of long-lived heavy colored particles, JHEP 09 (2008) 086 [hep-ph/0611322] [SPIRES].

[23] M. Kawasaki, K. Kohri and T. Moroi, Big-Bang nucleosynthesis and hadronic decay of long-lived massive particles, Phys. Rev. D 71 (2005) 083502 [astro-ph/0408426] [SPIRES].

[24] A. Arvanitaki, S. Dimopoulos, A. Pierce, S. Rajendran and J.G. Wacker, Stopping gluinos, Phys. Rev. D 76 (2007) 055007 [hep-ph/0506242] [SPIRES].

[25] Particle Data Group collaboration, C. Amsler et al., Review of particle physics, Phys. Lett. B 667 (2008) 1 [SPIRES].

[26] A. De Simone, M. Garny, A. Ibarra and C. Weniger, Supersymmetric leptogenesis with a light hidden sector, arXiv:1004.4890 [SPIRES]. 